\title{
Information Entropy and Information Granulation-based Uncertainty Measures in Incomplete Information Systems
}

\author{
Lin Sun ${ }^{1,2,3, *}$, Jiucheng $X u^{1,2}$ and Tianhe $X u^{1,2}$ \\ ${ }^{1}$ College of Computer and Information Engineering, Henan Normal University, 453007 Xinxiang, China \\ ${ }^{2}$ Engineering Technology Research Center for Computing Intelligence and Data Mining, Henan Province, China \\ ${ }^{3}$ International WIC Institute, Beijing University of Technology, 100124 Beijing, China
}

Received: 4 Sep. 2013, Revised: 7 Dec. 2013, Accepted: 8 Dec. 2013

Published online: 1 Jul. 2014

\begin{abstract}
In this paper, concepts of information entropy and information granulation-based uncertainty measures are introduced in incomplete information/decision systems, and based on maximal consistent block technique, some variants of information entropy and information granulation are presented to measure the discernibility ability of an incomplete information system. Then, some important properties of them are discussed. From these properties, it can be shown that these proposed measures provide important approaches to measure the uncertainty ability of different knowledge in incomplete information/decision systems. And relationships among these measures are established as well. These results will be very helpful for understanding the essence of knowledge content and uncertainty measures in incomplete information systems.
\end{abstract}

Keywords: Rough set, incomplete information system, information entropy, information granulation, uncertainty measure

\section{Introduction}

Rough set theory [1], as one of the important models of granular computing, is a popular mathematical framework to deal with inexact, uncertain or incomplete information $[2,3,4]$. The research has recently roused great interest in the theoretical and applicable fronts, such as pattern recognition, data mining, machine learning, decision support, and so on $[5,6,7,8]$. As one of the most important issues in rough set theory, uncertainty of a set and its measures have been widely studied $[9,10,11]$.

It is well known that the information entropy and the information granulation are two main approaches to measuring the uncertainty of an information system [12, 13]. The concept of entropy, originally defined by Shannon in 1948 for communication theory, gives a measure of the uncertainty about the structure of a system [14]. It has been a useful mechanism for characterizing uncertainty in a great diversity of modes and applications $[15,16,17,18]$. Shannon's entropy and its variants have been used to measure the uncertainty in rough set theory, and information interpretation of rough set theory was given in $[9,10,19]$. Beaubouef et al. [20] addressed information measures of uncertainty of rough sets and rough relation databases. Hernandez and Recasens [21] presented the formulae of joint entropy and conditional entropy. Miao and Wang [22] applied information entropy into rough set theory to measure the roughness of knowledge. However, Shannon's entropy is not a fuzzy entropy, and cannot measure the fuzziness in rough set theory. Liang et al. [23] presented a new information entropy to measure the fuzziness of rough set and rough classification. Mi et al. [24] gave a new fuzzy entropy and applied it to measure the fuzziness of a fuzzy-rough set-based partition. For rough sets in complete information systems, an improved uncertainty measure for rough sets was given in [25], which measured uncertainty of rough sets using excess entropy. In general, the information granulation represents the discernibility ability of an information/knowledge in information systems. As a powerful mechanism, granulation was first introduced by Zadeh [26]. It presents a more visual and easily understandable description for a partition on the universe. With regard to granular computing, many pieces

\footnotetext{
*Corresponding author e-mail: linsunok@gmail.com
} 
of nice work were accomplished $[27,28,29]$. Especially, closely associated with granular computing, several measures on knowledge in an information system were proposed and the relationships among these measures were discussed in [30]. Wierman [31] introduced the concept of granulation measure to measure the uncertainty of information. Liang and $\mathrm{Li}$ [10] investigated information granulation in complete information systems, which has been effectively applied in measuring attribute significance, feature selection, and decision-rule extracting, etc. To characterize the average measure of information granules, Liang and Qian [12] presented the definition of information granulation, and established the relationship between the information entropy and the information granulation. Therefore, it is mentioned that all these studies are dedicated to evaluating the uncertainty of a set in terms of the partition ability of knowledge. However, since the equivalence classes are only regarded as the unit of information granule of a complete information system, these measures above cannot be used to deal with incomplete information systems. In other words, the information systems considered in the above works are assumed to be complete. Researchers know well that the classical rough set theory is also presented for complete information systems. It is difficult to generalize the results in complete information systems to incomplete information systems. Therefore, it is necessary to extend and hybridize these measures to deal with incomplete data at present.

An incomplete information system, of which an incomplete decision system (sometimes called an incomplete decision table) can be considered as a special case, is an information system where some objects are missing data (null values) [32,33]. However, classical rough set theory is unsuitable for measuring the uncertainty and attribute reduction of incomplete information systems and incomplete decision systems. To address this issue, several interesting and meaningful extensions to equivalence relation have been proposed, such as tolerance relations $[9,11,12,30,32,33]$, neighborhood operators $[34,35]$, others $[36,37]$. In recent years, some new uncertainty measure theories and reduction methods have been developed, which are capable of reducing the attributes in incomplete information systems without preprocessing, and do not require changing the size of the original system. However, these methods are usually considered as extensions of classical rough set theory. These extended models are further analyzed and categorized according to the relations they employ. But until now, few studies have addressed the issue of measuring the uncertainty of knowledge in an incomplete decision system. For example, Huang et al. [38] introduced an informational entropy-based rough entropy measure to describe the uncertainty of knowledge roughness in incomplete information systems. Another rough entropy measure based on a generalized rough set covering reduction was also presented to incomplete information systems [39].
Qian et al. [40] proposed combination entropy and combination granulation with intuitionistic knowledge content characteristic in incomplete information systems, which can be used to measure the uncertainty of an incomplete information system, however, this measure has a complex mathematical form. Unfortunately, it should be noted that all the above mentioned measures of knowledge are not applicable for incomplete decision systems, and there are still several problems with these approaches from theoretical and practical viewpoint that motivate further developments in this issue. Therefore, it is desirable to present some uncertainty measures of knowledge to deal with incomplete data based on extended rough set models, and a further study on uncertainty measures suitable for an incomplete decision system is of both theoretical and practical importance. In this paper, the main objective is to construct some information entropy-based uncertainty measures and their corresponding information granulation in incomplete information/decision systems, and discuss their important properties. Furthermore, these proposed measures can provide important approaches to measure the uncertainty ability of different knowledge in incomplete decision systems, and then these results will be very helpful for understanding the essence of uncertainty measures in incomplete information systems.

The rest of this paper is organized as follows. Some preliminary concepts are briefly recalled in Section 2. In Section 3, concepts of information entropy, mutual information and conditional entropy in incomplete information/decision systems are introduced, and then based on maximal consistent block technique, a variant of information entropy is presented to an incomplete information system. Some of their important properties are induced, and then relationships among these measures are investigated. In Section 4, information granulation and conditional information granulation are introduced to measure the uncertainty of incomplete information/ decision systems, and a variant of information granulation with maximal consistent block is proposed to measure the discernibility ability of an information/knowledge in incomplete information systems. Then, several useful properties are derived, and relationships among them are established. Finally, Section 5 concludes the paper.

\section{Preliminaries}

In this section, we briefly review several basic concepts that are relevant to this paper. The detailed description of these theories can be found in $[1,10,12]$.

The notion of information system (IS) has been studied by many authors as a simple knowledge representation method. Formally, an information system is a quadruple $I S=(U, A, V, f)$, where $U$ is a finite nonempty set of objects indicating a given universe, $A$ is a finite nonempty set of attributes, $V$ is a union of attribute domains such that $V=\cup_{a \in A} V_{a}$ for $V_{a}$ denoting the value 
domain of attribute $a$, and $f: U \times A \rightarrow V$ is an information function which associates a unique value of each attribute with every object belonging to $U$, such that for any $a \in A$ and $u \in U, f(u, a) \in V_{a}$. Also, $(U, A, V, f)$ can be written more simply as $(U, A)$. With any subset $P \subseteq A$, there is an associated indiscernibility relation as follows: $\operatorname{IND}(P)$ $=\{(u, v) \in U \times U \mid \forall a \in P, f(u, a)=f(v, a)\}$. Since the indiscernibility relation $\operatorname{IND}(P)$ is also an equivalence relation on set $U$, it partitions $U$ into disjoint subsets (or equivalence classes). Let $U / I N D(P)$ denote the family of all equivalence classes of $\operatorname{IND}(P)$. For simplicity, $U / P$ will be written instead of $U / \operatorname{IND}(P)$. For every object $u \in U$, let $[u]_{P}$ denote the equivalence class of relation $\operatorname{IND}(P)$ that contains element $u$, called the equivalence class of $u$ under relation $I N D(P)$, i.e. $[u]_{P}=\{v \in U \mid(u, v)$ $\in I N D(P)\}$. Each equivalence class $[u]_{P}$ may be viewed as an information granule consisting of indistinguishable elements. The granulation structure induced by an equivalence relation is a partition of the universe.

It may happen that some of the attribute values of objects are missing, then these values are called missing values (or null values). These missing values can be represented by the set of all possible values for the attribute or equivalence by the domain of the attribute. To indicate such a situation, a distinguished value, the so-called null value is usually assigned to those attributes. If $V_{a}$ contains a null value for at least one attribute $a \in A$, then the information system $I S=(U, A)$ is called an incomplete information system (IIS), otherwise it is a complete information system $(C I S)$. Further on, the symbol $*$ denotes the missing value. If the value of an attribute $a$ is missing, then the real value must be from the set $V_{a}-\{*\}$. Any domain value different from $*$ will be called regular. Thus, an incomplete information system can be expressed as $I I S=(U, A), * \in \cup_{a \in A} V_{a}$. For any $P \subseteq A$, the subset $P$ determines a binary relation, denoted by $\operatorname{SIM}(P)=\{(u, v) \in U \times U \mid \forall a \in P, f(u, a)=f(v, a)$ or $f(u, a)=*$ or $f(v, a)=*\}$. In fact, it is easy to prove that $\operatorname{SIM}(P)$ is reflexive and symmetric, so it is a tolerance relation on $U$. The concept of a tolerance relation has a wide variety of applications in classification, which can be shown that $\operatorname{SIM}(P)=\cap_{a \in P} \operatorname{SIM}(\{a\})$.

Let $S_{P}(u)$ denote the set $\{v \in U \mid(u, v) \in \operatorname{SIM}(P)\}$. Generally, $S_{P}(u)$ denotes the maximal set of objects which are possibly indistinguishable by $P$ with object $u$. Let $U / \operatorname{SIM}(P)$ denote the family sets $\left\{S_{P}(u) \mid u \in U\right\}$, the classification or the knowledge induced by $P$. A member $S_{P}(u)$ from $U / \operatorname{SIM}(P)$ will be called a tolerance class or a granule of information. It should be noted that the tolerance classes in $U / \operatorname{SIM}(P)$ do not constitute a partition of $U$ in general. They constitute a cover of $U$, i.e., $S_{P}(u) \neq \emptyset$ for every $u \in U$, and $\cup_{u \in U} S_{P}(u)=U$. In particular, if $U / \operatorname{SIM}(P)=\omega=\left\{S_{P}(u)=\{u\} \mid u \in U\right\}$, it is called an identity relation, and if $U / \operatorname{SIM}(P)=\delta=$ $\left\{S_{P}(u)=U \mid u \in U\right\}$, it is called a universal relation.

Let IIS be an incomplete information system. Now, we define a partial relation $\preceq$ on $2^{A}$ as follows: we say that $P$ is finer than $Q$ (or $Q$ is coarser than $P$ ), denoted by $P \preceq Q$, if and only if $S_{P}\left(u_{i}\right) \subseteq S_{Q}\left(u_{i}\right)$ for any $i \in\{1,2, \cdots,|U|\}$. In fact, $P \prec Q \Leftrightarrow$ it follows that $S_{P}\left(u_{i}\right) \subseteq S_{Q}\left(u_{i}\right)$ for any $i \in\{1,2, \cdots,|U|\}$, and there exists $j \in\{1,2, \cdots,|U|\}$ such that $S_{P}\left(u_{j}\right) \subset S_{Q}\left(u_{j}\right)$.

An incomplete information system $I I S=(U, C \cup D)$ is called an incomplete decision system (IDS) if condition attributes and decision attributes are distinguished, where $C$ is the condition attribute set and $D$ is the decision attribute set with $C \cap D=\emptyset$. That is, an incomplete decision system is a special case of an incomplete information system.

As we know, tolerance classes are not the minimal units for describing knowledge or information in an incomplete information system or an incomplete decision system. Let IIS be an incomplete information system, $P \subseteq A$ an attribute set and $X \subseteq U$ a subset of objects. We say that $X$ is consistent with respect to $P$ if $(u, v) \in \operatorname{SIM}(P)$ for any $u, v \in X$. If there does not exist a subset $Y \subseteq U$ such that $X \subset Y$, and $Y$ is consistent with respect to $P$, then $X$ is called a maximal consistent block of $P$. Obviously, in a maximal consistent block, all objects are not indiscernible with available information provided by a similarity relation [32]. Thus, the set of all maximal consistent blocks determined by $P \subseteq A$ is denoted by $C_{P}$, and the set of all maximal consistent blocks of $P$ which includes some object $u \in U$ is denoted as $C_{P}(u)$. It is obvious that $X \in C_{P}$ if and only if $X=\cap_{u \in X} S_{p}(u)$. Note that the set of all maximal consistent blocks $C_{P}$ degenerates into the partition $U / P$ induced by attribute set $P$ in a complete information system, i.e., $C_{P}=U / P$.

\section{Information entropy-based uncertainty measures in incomplete information systems}

In this section, the concept of information entropy has been introduced in complete information systems, and it is extended to measure the uncertainty of knowledge in incomplete information systems. Then, some information entropy-based uncertainty measures are presented to incomplete information systems and incomplete decision systems. The properties of these uncertainty measures are discussed respectively, and the relationships among them are established as well.

\subsection{Information entropy and conditional entropy in incomplete information systems}

Entropy is always used to measure the out-of-order degree of a system, then Shannon introduced the concept of entropy in physics to information entropy for measuring uncertainty of the structure of a system $[9,12$, 14]. However, Shannon's entropy cannot measure the fuzziness in rough set theory. To address this issue, Liang et al. [23] proposed the new information entropy to measure uncertainty in complete information systems, 
which can be used to measure both uncertainty and fuzziness in rough set theory. The following definition gives the depiction of the information entropy in a complete information system.

Let $C I S$ be a complete information system and $U / A=$ $\left\{R_{1}, R_{2}, \cdots, R_{m}\right\}$. Information entropy of knowledge $A$ for rough set theory is denoted by

$$
E(A)=\sum_{i=1}^{m} \frac{\left|R_{i}\right|}{|U|} \frac{\left|R_{i}^{c}\right|}{|U|}=\sum_{i=1}^{m} \frac{\left|R_{i}\right|}{|U|}\left(1-\frac{\left|R_{i}\right|}{|U|}\right)
$$

where $R_{i}^{c}$ is the complement of $R_{i}$, i.e., $R_{i}^{c}=U-R_{i}, \frac{\left|R_{i}\right|}{|U|}$ represents the probability of equivalence class $R_{i}$ within the universe $U$, and $\frac{\left|R_{i}^{c}\right|}{|U|}$ represents the probability of the complement set of $R_{i}$ within the universe $U$.

Proposition 3.1. Let $C I S$ be a complete information system and $P, Q \subseteq A$. If $Q \subseteq P$, then $E(Q) \leq E(P)$.

Proof. Since $Q \subseteq P$, it follows that $P \preceq Q$, i.e., $P \prec Q$ and $P=Q$. If $P \prec Q$, from Theorem 12 in [12], one has that $E(Q)<E(P)$. If $P=Q$, it is obvious that $E(Q)=E(P)$. Hence, if $Q \subseteq P, E(Q) \leq E(P)$. This completes the proof.

When we do not need to distinguish complete information systems from incomplete information systems, an information in an $I S$ can be represented as the vector $K(A)=\left\{S_{A}\left(u_{1}\right), S_{A}\left(u_{2}\right), \cdots, S_{A}\left(u_{|U|}\right)\right\} \quad$ [10]. Let $C I S$ be a complete information system and $U / A=\left\{X_{1}, X_{2}, \quad \cdots, X_{m}\right\}$. If the set $X_{i}=\left\{u_{i 1}, u_{i 2}, \cdots, u_{i s_{i}}\right\}$, where $\left|X_{i}\right|=s_{i}$, and $\sum_{i=1}^{m} s_{i}=|U|$, then the relationship between $K(A)$ and $U / A$ is as follows: $X_{i}=S_{A}\left(u_{i 1}\right)=S_{A}\left(u_{i 2}\right)=\cdots=S_{A}\left(u_{i s_{i}}\right)$, i.e., $\left|X_{i}\right|=\left|S_{A}\left(u_{i 1}\right)\right|=\left|S_{A}\left(u_{i 2}\right)\right|=\cdots=\left|S_{A}\left(u_{i s_{i}}\right)\right|$. Therefore, we have that $E(A)=\sum_{i=1}^{m} \frac{\left|X_{i}\right|}{|U|}\left(1-\frac{\left|X_{i}\right|}{|U|}\right)=\sum_{i=1}^{m}\left(\frac{1}{|U|}(1-\right.$ $\left.\frac{\left|S_{A}\left(u_{i 1}\right)\right|}{|U|}\right)+\frac{1}{|U|}\left(1 \quad-\quad \frac{\left|S_{A}\left(u_{i 2}\right)\right|}{|U|}\right)+\cdots+$ $\left.\frac{1}{|U|}\left(1-\frac{\left|S_{A}\left(u_{i s_{i}}\right)\right|}{|U|}\right)\right)=\sum_{i=1}^{|U|} \frac{1}{|U|}\left(1-\frac{\left|S_{A}\left(u_{i}\right)\right|}{|U|}\right)$.

Definition 3.1. Let $I I S$ be an incomplete information system with $P \subseteq A, U / \operatorname{SIM}(P)=\left\{S_{P}\left(u_{1}\right), S_{P}\left(u_{2}\right), \cdots\right.$, $\left.S_{P}\left(u_{|U|}\right)\right\}$. Information entropy of knowledge $P$ is defined as

$$
I E(P)=\sum_{i=1}^{|U|} \frac{1}{|U|}\left(1-\frac{\left|S_{P}\left(u_{i}\right)\right|}{|U|}\right) .
$$

Property 3.1. Let $I I S$ be an incomplete information system with $P \subseteq A$. The minimum of information entropy of knowledge $P$ is 0 . This value is achieved only by the $U / \operatorname{SIM}(P)=\delta$. The maximum of information entropy of knowledge $P$ is $1-\frac{1}{|U|}$. This value is achieved only by the $U / \operatorname{SIM}(P)=\omega$. Obviously, for an IIS, we have that $0 \leq$ $I E(A) \leq 1-\frac{1}{|U|}$.

It is noted that Shannon's entropy and its variants have been applied to measure the uncertainty in complete information systems [39]. However, some of these entropies cannot be used in incomplete information systems. Mi et al. [24] and Yu et al. [41] investigated how to measure the uncertainty of a partition-based fuzzy rough set and that of a fuzzy information system respectively. However, these measures only deal with the fuzziness and also cannot be used for incomplete information systems. Unlike Shannon's entropy, the proposed information entropy of knowledge can measure not only randomness and fuzziness in an incomplete information system, but also fuzziness of a rough set and a rough classification [12]. Therefore, the above information entropy of knowledge may be better for calculating the uncertainty in incomplete information systems.

Proposition 3.2. Let $I I S$ be an incomplete information system and $P, Q \subseteq A$. If $P \prec Q$, then $I E(Q)<I E(P)$.

Proof. Let $U / \operatorname{SIM}(P)=\left\{S_{P}\left(u_{1}\right), S_{P}\left(u_{2}\right), \cdots, S_{P}\left(u_{|U|}\right)\right\}$ and $U / \operatorname{SIM}(Q)=\left\{S_{Q}\left(u_{1}\right), S_{Q}\left(u_{2}\right), \cdots, S_{Q}\left(u_{|U|}\right)\right\}$. Since $P \prec Q$, it follows that $S_{P}\left(u_{i}\right) \subseteq S_{Q}\left(u_{i}\right)$ for any $u_{i} \in U$, i.e., $\left|S_{P}\left(u_{i}\right)\right| \leq\left|S_{Q}\left(u_{i}\right)\right|$, and there exists $j \in\{1,2, \cdots,|U|\}$ such that $S_{P}\left(u_{j}\right) \subset S_{Q}\left(u_{j}\right)$, i.e., $\left|S_{P}\left(u_{j}\right)\right|<\left|S_{Q}\left(u_{j}\right)\right|$. Hence, it is obvious that $\frac{\left|S_{P}\left(u_{i}\right)\right|}{|U|} \leq \frac{\left|S_{Q}\left(u_{i}\right)\right|}{|U|} \Rightarrow \frac{1}{|U|}(1-$ $\left.\frac{\left|S_{Q}\left(u_{i}\right)\right|}{|U|}\right) \leq \frac{1}{|U|}\left(1-\frac{\left|S_{P}\left(u_{i}\right)\right|}{|U|}\right) \Rightarrow \sum_{i=1, u_{i} \neq u_{j}}^{|U|} \frac{1}{|U|}\left(1-\frac{\left|S_{Q}\left(u_{i}\right)\right|}{|U|}\right)+$ $\frac{1}{|U|}\left(1-\frac{\left|S_{Q}\left(u_{j}\right)\right|}{|U|}\right)<\sum_{i=1, u_{i} \neq u_{j}}^{|U|} \frac{1}{|U|}\left(1-\frac{\left|S_{P}\left(u_{i}\right)\right|}{|U|}\right)+\frac{1}{|U|}(1-$ $\left.\frac{\left|S_{P}\left(u_{j}\right)\right|}{|U|}\right) \Rightarrow \sum_{i=1}^{|U|} \frac{1}{|U|}\left(1-\frac{\left|S_{Q}\left(u_{i}\right)\right|}{|U|}\right)<\sum_{i=1}^{|U|} \frac{1}{|U|}\left(1-\frac{\left|S_{P}\left(u_{i}\right)\right|}{|U|}\right)$, i.e., $I E(Q)<I E(P)$. This completes the proof.

Proposition 3.2 states that the information entropy of knowledge monotonically increases as tolerance classes become smaller through finer classification with the increase of attributes in knowledge, which is consistent with the situation of information entropy in complete information systems from Proposition 3.1.

Proposition 3.3. Let $I I S$ be an incomplete information system, $P, Q \subseteq A$. If $\operatorname{SIM}(P)=\operatorname{SIM}(Q), \operatorname{IE}(P)=\operatorname{IE}(Q)$.

Proof. Suppose that $U=\left\{u_{1}, u_{2}, \cdots, u_{|U|}\right\}$, and $P, Q \subseteq A$. If $\operatorname{SIM}(P)=\operatorname{SIM}(Q)$, it follows that $S_{Q}\left(u_{i}\right)=S_{P}\left(u_{i}\right)$ for any $u_{i} \in U$. Thus, one has that $\sum_{i=1}^{|U|} \frac{1}{|U|}\left(1-\frac{\left|S_{P}\left(u_{i}\right)\right|}{|U|}\right)=$ $\sum_{i=1}^{|U|} \frac{1}{|U|}\left(1-\frac{\left|S_{Q}\left(u_{i}\right)\right|}{|U|}\right)$, i.e., $\operatorname{IE}(P)=\operatorname{IE}(Q)$. Therefore, the proof is completed.

Proposition 3.3 shows that the information entropy of two knowledge having the same tolerance relations is equal in incomplete information systems. Note that the reverse relation of Proposition 3.3 cannot be established.

Proposition 3.4. Let $I I S$ be an incomplete information system and $P \subseteq Q \subseteq A$. If $\operatorname{IE}(P)=\operatorname{IE}(Q)$, then $\operatorname{SIM}(P)$ $=\operatorname{SIM}(Q)$.

Proof. Suppose that $U=\left\{u_{1}, u_{2}, \cdots, u_{|U|}\right\}$, and $P \subseteq Q \subseteq$ $A$, it follows that for any $i \in\{1,2, \cdots,|U|\}$, we have $S_{Q}\left(u_{i}\right)$ $\subseteq S_{P}\left(u_{i}\right)$. If $I E(P)=I E(Q)$, it is obvious from Definition 3.1 that $S_{P}\left(u_{i}\right)=S_{Q}\left(u_{i}\right)$ for any $u_{i} \in U$. Hence, $\operatorname{SIM}(P)=$ $\operatorname{SIM}(Q)$ holds. This completes the proof.

Proposition 3.4 states that for two knowledge having a conclusion relation, if their information entropies in 
incomplete information systems are equal, then they have the same tolerance relations.

Proposition 3.5. Let $I I S$ be an incomplete information system and $P \subseteq A . I E(P)=I E(A)$ if and only if $U / \operatorname{SIM}(P)=U / \operatorname{SIM}(A)$.

Proof. $\Rightarrow$ Suppose that $P \subseteq A$, and $I E(P)=I E(A)$, it follows from Proposition 3.4 that $\operatorname{SIM}(P)=\operatorname{SIM}(A)$. Thus, $S_{P}\left(u_{i}\right)=\left\{u_{j} \in U \mid\left(u_{i}, u_{j}\right) \in \operatorname{SIM}(P)\right\}=\left\{u_{k} \in U \mid\right.$ $\left.\left(u_{i}, u_{k}\right) \in \operatorname{SIM}(A)\right\}=S_{A}\left(u_{i}\right)$ for any $i, j$ and $k \in\{1,2, \cdots$, $|U|\}$. One has that $\cup\left\{S_{P}\left(u_{i}\right) \mid u_{i} \in U\right\}=\cup\left\{S_{A}\left(u_{i}\right) \mid u_{i} \in U\right\}$ for any $i \in\{1,2, \cdots,|U|\}$, i.e., $U / \operatorname{SIM}(P)=U / \operatorname{SIM}(A)$. $\Leftarrow$ If $U / \operatorname{SIM}(P)=U / \operatorname{SIM}(A)$, that is, one has that $S_{P}\left(u_{i}\right)$ $=S_{A}\left(u_{i}\right)$ for any $i \in\{1,2, \cdots,|U|\}$ such that $\operatorname{SIM}(P)=$ $\operatorname{SIM}(A)$. Thus, it follows from Proposition 3.3 that $I E(P)$ $=I E(A)$. This completes the proof.

To investigate the information entropy of new knowledge composed of two given knowledge with the same universe in an incomplete information system, we introduce Lemma 1 in [9] as follows.

Lemma 3.1. Let IIS be an incomplete information system and $P, Q \subseteq A$. Then the following properties hold

(1) $\operatorname{SIM}(\bar{P}) \cap \operatorname{SIM}(Q)=\operatorname{SIM}(P \cup Q)$;

(2) $S_{P}(u) \cap S_{Q}(u)=S_{P \cup Q}(u)$ for any $u \in U$;

(3) $U / \operatorname{SIM}(P) \cap U / \operatorname{SIM}(Q)=U / \operatorname{SIM}(P \cup Q)$;

(4) $\bigcup_{i=1}^{i=|U|} \bigcup_{j=1}^{j=|U|}\left\{S_{P}\left(u_{i}\right) \cap S_{Q}\left(u_{j}\right)\right\}=\bigcup_{i=1}^{i=|U|}\left\{S_{P \cup Q}\left(u_{i}\right)\right\}$

$=\bigcup_{i=1}^{i=|U|}\left\{S_{P}\left(u_{i}\right) \cap S_{Q}\left(u_{i}\right)\right\}$.

Definition 3.2. Let $I I S_{1}=(U, P), I I S_{2}=(U, Q)$ be two incomplete information systems. Joint information entropy of $P$ and $Q$ is defined as

$$
\begin{aligned}
I E(P \cup Q) & =\sum_{i=1}^{|U|} \sum_{j=1}^{|U|} \frac{1}{|U|}\left(1-\frac{\left|S_{P}\left(u_{i}\right) \cap S_{Q}\left(u_{j}\right)\right|}{|U|}\right) \\
& =\sum_{i=1}^{|U|} \frac{1}{|U|}\left(1-\frac{\left|S_{P}\left(u_{i}\right) \cap S_{Q}\left(u_{i}\right)\right|}{|U|}\right) .
\end{aligned}
$$

Definition 3.2 denotes the joint information entropy of new information system composed of two given information systems with the same universe in incomplete information systems.

Proposition 3.6. Let $I I S$ be an incomplete information system and $P, Q \subseteq A$. Then the following properties hold

(1) $I E(P) \leq I E(P \cup Q), I E(Q) \leq I E(P \cup Q)$;

(2) If $P \prec \bar{Q}$, then $I E(P \cup Q)=\bar{I} E(P)$.

Proof. It is achieved by Definition 3.2 and Proposition 3.2.

Definition 3.3. Let $I I S$ be an incomplete information system with $P, Q \subseteq A$. Conditional entropy of $Q$ with respect to $P$ is defined as

$$
I E(Q \mid P)=\sum_{i=1}^{|U|} \frac{\left|S_{P}\left(u_{i}\right)\right|-\left|S_{P}\left(u_{i}\right) \cap S_{Q}\left(u_{i}\right)\right|}{|U|^{2}}
$$

Definition 3.4. Let $I I S$ be an incomplete information system with $P, Q \subseteq A$. Mutual information between $P$ and $Q$ is defined as

$$
I E(P ; Q)=I E(P)+I E(Q)-I E(P \cup Q) .
$$

Here, the following propositions will establish the relationships among the information entropy, the joint information entropy, the conditional entropy and the mutual information in an incomplete information system.

Proposition 3.7. Let $I I S$ be an incomplete information system with $P, Q \subseteq A . I E(Q \mid P)=I E(P \cup Q)-I E(P)$.

Proof. It follows immediately from Definition 3.3 that

$$
\begin{aligned}
& \operatorname{IE}(Q \mid P) \\
= & \sum_{i=1}^{|U|} \frac{\left|S_{P}\left(u_{i}\right)\right|}{|U|^{2}}-\sum_{i=1}^{|U|} \frac{\left|S_{P}\left(u_{i}\right) \cap S_{Q}\left(u_{i}\right)\right|}{|U|^{2}}+\sum_{i=1}^{|U|} \frac{1}{|U|}-\sum_{i=1}^{|U|} \frac{1}{|U|} \\
= & \sum_{i=1}^{|U|}\left(\frac{1}{|U|}-\frac{\left|S_{P}\left(u_{i}\right) \cap S_{Q}\left(u_{i}\right)\right|}{|U|^{2}}\right)-\sum_{i=1}^{|U|}\left(\frac{1}{|U|}-\frac{\left|S_{P}\left(u_{i}\right)\right|}{|U|^{2}}\right) \\
= & \sum_{i=1}^{|U|} \frac{1}{|U|}\left(1-\frac{\left|S_{P}\left(u_{i}\right) \cap S_{Q}\left(u_{i}\right)\right|}{|U|}\right)-\sum_{i=1}^{|U|} \frac{1}{|U|}\left(1-\frac{\left|S_{P}\left(u_{i}\right)\right|}{|U|}\right) \\
= & I E(P \cup Q)-I E(P) .
\end{aligned}
$$

Proposition 3.8. Let $I I S$ be an incomplete information system and $P, Q \subseteq A$. Then $I E(P ; Q)=I E(P)-I E(P \mid Q)$ $=I E(Q)-I E(Q \mid P)$.

Proof. From Definition 3.4 and Proposition 3.7, we have that $I E(P ; Q)=I E(P)+I E(Q)-I E(P \cup Q)=I E(Q)-$ $(I E(P \cup Q)-I E(P))=I E(Q)-I E(Q \mid P)$. Similarly, the equation $I E(P ; Q)=I E(P)-I E(P \mid Q)$ can be proved. This completes the proof.

Note that these equations cannot be satisfied by some existing measures in incomplete information systems. Furthermore, these relationships will be helpful for understanding the essence of the knowledge content and the uncertainty in incomplete information systems.

Proposition 3.9. Let $I I S$ be an incomplete information system and $P, Q \subseteq A$. IE $(Q \mid P)=0$ if and only if $P \preceq Q$.

Proof. $\Rightarrow$ Suppose $I E(Q \mid P)=0$, we need to prove $P \preceq$ $Q$. If $P \preceq Q$ does not hold, then there exists some $u_{j} \in \bar{U}$ such that $S_{P}\left(u_{j}\right) \subseteq S_{Q}\left(u_{j}\right)$ does not hold, i.e., $1 \leq \mid S_{P}\left(u_{j}\right) \cap$ $S_{Q}\left(u_{j}\right)|<| S_{P}\left(u_{j}\right) \mid$. Therefore, we have that

$$
\begin{aligned}
I E(Q \mid P)= & \sum_{i=1, u_{i} \neq u_{j}}^{|U|} \frac{\left|S_{P}\left(u_{i}\right)\right|-\left|S_{P}\left(u_{i}\right) \cap S_{Q}\left(u_{i}\right)\right|}{|U|^{2}}+ \\
& \frac{\left|S_{P}\left(u_{j}\right)\right|-\left|S_{P}\left(u_{j}\right) \cap S_{Q}\left(u_{j}\right)\right|}{|U|^{2}} \\
\geq & \frac{\left|S_{P}\left(u_{j}\right)\right|-\left|S_{P}\left(u_{j}\right) \cap S_{Q}\left(u_{j}\right)\right|}{|U|^{2}} \\
> & \frac{\left|S_{P}\left(u_{j}\right)\right|-\left|S_{P}\left(u_{j}\right)\right|}{|U|^{2}}=0,
\end{aligned}
$$


i.e., $I E(Q \mid P)>0$. This yields a contradiction. Thus, $P \preceq Q$ holds.

$\Leftarrow$ Suppose $P \preceq Q$, then, for any $u_{i} \in U$, it follows that $S_{P}\left(u_{i}\right) \subseteq S_{Q}\left(u_{i}\right)$, i.e., $S_{P}\left(u_{i}\right) \cap S_{Q}\left(u_{i}\right)=S_{P}\left(u_{i}\right)$. Hence, we can obtain that $I E(Q \mid P)=\sum_{i=1}^{|U|} \frac{\left|S_{P}\left(u_{i}\right)\right|-\left|S_{P}\left(u_{i}\right)\right|}{|U|^{2}}=0$. This completes the proof.

Proposition 3.9 illustrates that in the same universe, a knowledge cannot provide the system any additional uncertainty (classification information) if it is coarser than the original knowledge in incomplete information systems. It is noted that in a given decision information system, this decision attribute usually generates the corresponding partition or covering. Thus, to reveal the relationship between the condition attributes and the decision attribute, we present following definitions and relative properties.

Definition 3.5. Let $I D S$ be an incomplete decision system with $P \subseteq C, U / \operatorname{SIM}(P)=\left\{S_{P}\left(u_{1}\right), S_{P}\left(u_{2}\right), \cdots, S_{P}\left(u_{|U|}\right)\right\}$, and $U / \operatorname{SIM}(D)=\left\{S_{D}\left(u_{1}\right), S_{D}\left(u_{2}\right), \cdots, S_{D}\left(u_{|U|}\right)\right\}$. The conditional entropy of $D$ with respect to $P$ is defined as

$$
I E(D \mid P)=\sum_{i=1}^{|U|} \frac{\left|S_{P}\left(u_{i}\right)\right|-\left|S_{P}\left(u_{i}\right) \cap S_{D}\left(u_{i}\right)\right|}{|U|^{2}} .
$$

Property 3.2. Let $I D S$ be an incomplete decision system with $P \subseteq C$. If $U / \operatorname{SIM}(P)=\omega$, then $I E(D \mid P)$ achieves the minimum value 0 . If $U / \operatorname{SIM}(P)=\delta$ and $U / \operatorname{SIM}(D)=\omega$, then $I E(D \mid P)$ achieves the maximum value $1-\frac{1}{|U|}$. When $\operatorname{SIM}(C)$ is a tolerance relation, $0 \leq \operatorname{IE}(D \mid C) \leq 1-\frac{1}{|U|}$.

Proposition 3.10. Let $I D S$ be an incomplete decision system, $P, Q \subseteq C$. If $P \preceq Q$, then $I E(Q \mid D) \leq I E(P \mid D)$.

Proof. Since $P \preceq Q$, it follows that $S_{P}\left(u_{i}\right) \subseteq S_{Q}\left(u_{i}\right)$, $\left|S_{P}\left(u_{i}\right)\right| \leq\left|S_{Q}\left(u_{i}\right)\right|$, and $\left|S_{D}\left(u_{i}\right) \cap S_{P}\left(u_{i}\right)\right| \leq \mid S_{D}\left(u_{i}\right) \cap$ $S_{Q}\left(u_{i}\right) \mid$ for any $u_{i} \in U$ and $S_{D}\left(u_{i}\right) \in U / \operatorname{SIM}(D)$. From Definition 3.3, we can obtain that

$$
\begin{aligned}
I E(Q \mid D) & =\sum_{i=1}^{|U|} \frac{\left|S_{D}\left(u_{i}\right)\right|-\left|S_{D}\left(u_{i}\right) \cap S_{Q}\left(u_{i}\right)\right|}{|U|^{2}} \\
& \leq \sum_{i=1}^{|U|} \frac{\left|S_{D}\left(u_{i}\right)\right|-\left|S_{D}\left(u_{i}\right) \cap S_{P}\left(u_{i}\right)\right|}{|U|^{2}}=\operatorname{IE}(P \mid D)(9)
\end{aligned}
$$

i.e., $I E(Q \mid D) \leq I E(P \mid D)$. This completes the proof.

Proposition 3.10 indicates that the finer the condition knowledge is, the more classification information it can provide to an apriori knowledge (target decision) in incomplete decision systems. However, it is noted that the reverse relation of Proposition 3.10 cannot be established in general. In the following, the performance of this relation in an incomplete decision system is shown through an illustrative example.

Example 3.1. Consider an incomplete decision system about several cars shown in Table 1, where $C=$
Table 1: An incomplete decision system about cars

\begin{tabular}{cccccc}
\hline car & $P$ & $M$ & $S$ & $X$ & $D$ \\
\hline 1 & High & Low & Full & Low & Good \\
2 & Low & $*$ & Full & Low & Good \\
3 & $*$ & $*$ & Compact & Low & Poor \\
4 & High & $*$ & Full & High & Good \\
5 & $*$ & $*$ & Full & High & Excellent \\
6 & Low & High & Full & $*$ & Good \\
\hline
\end{tabular}

$\{$ Price, Mileage, Size, Max - Speed $\}=\{P, M, S, X\}$ and $D=\{$ Acceleration $\}$.

Assume that $U / \operatorname{SIM}(\{P, M\})=\{\{1,3,4,5\},\{2,3,5,6\}$, $\{1,2,3,4,5,6\},\{1,3,4,5\},\{1,2,3,4,5,6\},\{2,3,5,6\}\}$,

$U / \operatorname{SIM}(\{S, X\})=\{\{1,2,6\},\{1,2,6\},\{3\},\{4,5,6\},\{4,5,6\}$, $\{1,2,4,5,6\}\}$, and $U / \operatorname{SIM}(D)=\{\{1,2,4,6\},\{1,2,4,6\}$, $\{3\},\{1,2,4,6\},\{5\},\{1,2,4,6\}\}$. It is easily computed that $I E(\{P, M\} \mid D)=\frac{4-2+4-2+1-1+4-2+1-1+4-2}{6^{2}}=\frac{2}{9}$, and $I E(\{S, X\} \mid D)=\frac{4-3+4-3+1-1+4-2+1-1+4-4}{6^{2}}=\frac{1}{9}$, i.e., $\operatorname{IE}(\{P, M\} \mid D)>\operatorname{IE}(\{S, X\} \mid D)$. However, $\{P, M\} \preceq$ $\{S, X\}$ cannot be satisfied in fact.

As we know, conditional entropy can be usually used to define the significance of an attribute set of decision systems in practice. In what follows, we further discuss the relationship between two conditional entropies in incomplete decision systems.

Proposition 3.11. Let $I D S$ be an incomplete decision system with $P, Q \subseteq C$. If $P \preceq Q, I E(D \mid P) \leq I E(D \mid Q)$.

Proof. Since $P \preceq Q$, it follows that $S_{P}\left(u_{i}\right) \subseteq S_{Q}\left(u_{i}\right)$, $\left|S_{P}\left(u_{i}\right)\right| \leq\left|S_{Q}\left(u_{i}\right)\right|$, and then $S_{P}\left(u_{i}\right) \cap S_{D}\left(u_{i}\right) \subseteq S_{Q}\left(u_{i}\right) \cap$ $S_{D}\left(u_{i}\right)$ for any $u_{i} \in U$, and $S_{D}\left(u_{i}\right) \in U / \operatorname{SIM}(D)$. From Definition 3.5, we have that

$$
\begin{aligned}
& \operatorname{IE}(D \mid Q)-I E(D \mid P) \\
= & \sum_{i=1}^{|U|}\left(\frac{\left|S_{Q}\left(u_{i}\right)\right|-\left|S_{P}\left(u_{i}\right)\right|}{|U|^{2}}-\right. \\
& \left.\frac{\left|S_{Q}\left(u_{i}\right) \cap S_{D}\left(u_{i}\right)\right|-\left|S_{P}\left(u_{i}\right) \cap S_{D}\left(u_{i}\right)\right|}{|U|^{2}}\right) \\
= & \sum_{i=1}^{|U|} \frac{\left|S_{Q}\left(u_{i}\right)\right|-\left|S_{P}\left(u_{i}\right)\right|-\left|S_{D}\left(u_{i}\right) \cap\left(S_{Q}\left(u_{i}\right)-S_{P}\left(u_{i}\right)\right)\right|}{|U|^{2}} \\
\geq & 0,
\end{aligned}
$$

i.e., $I E(D \mid P) \leq I E(D \mid Q)$. Thus, it is obvious that $I E(D \mid P)$ $=I E(D \mid Q)$ if and only if $\left\{S_{Q}\left(u_{i}\right)-S_{P}\left(u_{i}\right)\right\} \subseteq S_{D}\left(u_{i}\right)$ for any $u_{i} \in U$. This completes the proof.

Proposition 3.11 shows that the coarser the condition knowledge is, the more classification information it can preserve with respect to a target decision. In other words, in the $I D S$, if $I E(D \mid P) \leq I E(D \mid Q)$, one says that the attribute set $P$ is more significant than the attribute set $Q$ with respect to the target decision $D$.

Proposition 3.12. Let IDS be an incomplete decision system, $P, Q \subseteq C$. If $P \preceq Q \preceq D, I E(D \mid P)=I E(D \mid Q)=0$. 
Proof. Since $P \preceq Q \preceq D$, it follows that $S_{P}\left(u_{i}\right) \subseteq S_{Q}\left(u_{i}\right)$ $\subseteq S_{D}\left(u_{i}\right), S_{P}\left(u_{i}\right) \cap S_{D}\left(u_{i}\right)=S_{P}\left(u_{i}\right)$, and $S_{Q}\left(u_{i}\right) \cap S_{D}\left(u_{i}\right)$ $=S_{Q}\left(u_{i}\right)$ for any $u_{i} \in U$, and $S_{D}\left(u_{i}\right) \in U / \operatorname{SIM}(D)$. From Definition 3.5, we have $I E(D \mid P)=\sum_{i=1}^{|U|} \frac{\left|S_{P}\left(u_{i}\right)\right|-\left|S_{P}\left(u_{i}\right)\right|}{|U|^{2}}=0$. Similarly, the equation $I E(D \mid Q)=0$ can be proved easily. This completes the proof.

Until now, most of the existing uncertainty measures cannot be used in incomplete decision systems. According to the properties mentioned and the above corresponding discussions, it is known that the conditional entropy proposed above can well characterize the uncertainty of knowledge in incomplete decision systems. So far, however, these uncertainty measures and the relationships among them above have not been reported in incomplete information systems and incomplete decision systems. In fact, given any binary relation, one can induce a cover of the universe and determine a particular information system. Furthermore, through using the idea of information view, we may use the information entropy or its variants to measure the uncertainty of the information systems induced by a given binary relation. Thus, the information entropy and the conditional entropy can not only characterize the uncertainty of an incomplete information system, but also measure those of other kinds of information systems.

\subsection{Information entropy with maximal consistent block in incomplete information systems}

Because the maximal consistent block technique can describe the minimal units for knowledge or information in incomplete information systems $[32,40]$, we now introduce a variant of the proposed information entropy with maximal consistent block to measure the uncertainty of incomplete information systems.

Let IIS be an incomplete information system, then the maximal consistent blocks are denoted by $C_{A}=$ $\left\{A^{1}, A^{2}, \cdots, A^{m}\right\}$ induced by $A$. Of particular interest is the discrete classification $C_{A}=\omega=\{\{u\} \mid u \in U\}$ and the discrete classification $C_{A}=\delta=\{U \mid u \in U\}$, or just $\delta$ and $\omega$ if there is no confusion as to the domain set [40].

Definition 3.6. Let $I I S$ be an incomplete information system, $P \subseteq A$, and $C_{P}=\left\{P^{1}, P^{2}, \cdots, P^{m}\right\}$. Information entropy of knowledge $P$ for the maximal consistent blocks $C_{P}$ is defined as

$$
I E\left(C_{P}\right)=\sum_{i=1}^{m} \frac{1}{|m|}\left(1-\frac{\left|P^{i}\right|}{|U|}\right),
$$

where $\frac{\left|P^{i}\right|}{|U|}$ represents the probability of maximal consistent block $P^{i}$ within the universe $U$.

Property 3.3. Let IIS be an incomplete information system with $P \subseteq A$. If $C_{P}=\delta$, then the information entropy of knowledge $P$ achieves the minimum value
$I E\left(C_{P}\right)=0$. If $C_{P}=\omega$, then the information entropy of knowledge $P$ achieves the maximum value $I E\left(C_{P}\right)$ $=1-\frac{1}{\mid U}$. Obviously, for an $I I S$, we have that $0 \leq I E\left(C_{A}\right)$ $\leq 1-\frac{1}{U}$ holds.

Let IIS be an incomplete information system, $P, Q \subseteq A$, $C_{P}=\left\{P^{1}, P^{2}, \cdots, P^{m}\right\}$, and $C_{Q}=\left\{Q^{1}, Q^{2}, \cdots, Q^{n}\right\}$. Then we define a partial order $\preceq^{\prime}$ (or $\succeq^{\prime}$ ) in the view of maximal consistent block nature of knowledge on $U$ as follows: $P$ is finer than $Q$ (or $Q$ is coarser than $P$ ), denoted by $P \preceq^{\prime} Q$ (or $Q \succeq^{\prime} P$ ), if and only if for every $P^{i} \in C_{P}$, there exists $Q^{j} \in C_{Q}$ such that $P^{i} \subseteq Q^{j}$. Thus, $P \prec^{\prime} Q$, for every $P^{i} \in$ $C_{P}$, there exists $Q^{j} \in C_{Q}$ such that $P^{i} \subseteq Q^{j}$, and for some $P^{i 0} \in C_{P}$, there exists $Q^{j 0} \in C_{Q}$ such that $P^{i 0} \subset Q^{j 0}$.

Proposition 3.13. Let $I I S$ be an incomplete information system, $P, Q \subseteq A, C_{P}=\left\{P^{1}, P^{2}, \cdots, P^{m}\right\}$, and $C_{Q}=\left\{Q^{1}\right.$, $\left.Q^{2}, \cdots, Q^{n}\right\}$. If $P \prec^{\prime} Q$, then $\operatorname{IE}\left(C_{Q}\right)<\operatorname{IE}\left(C_{P}\right)$.

Proof. Since $P \prec^{\prime} Q$, it follows that for every $P^{i} \in C_{P}$, there exists $Q^{j} \in C_{Q}$ such that $P^{i} \subseteq Q^{j}$, and for some $P^{i 0} \in C_{P}$, there exists $Q^{j 0} \in C_{Q}$ such that $P^{i 0} \subset Q^{j 0}$ and $m>n$. Then, for $Q^{j 0} \in C_{Q}$, there exists $\left\{P^{i 1}, P^{i 2}, \cdots, P^{i s}\right\}$ $\left(P^{i k} \in C_{P}, k=\{1,2, \cdots, s\}\right)$ such that each $P^{i k} \subseteq Q^{j 0}$, where $P^{i 0} \in\left\{P^{i 1}, P^{i 2}, \cdots, P^{i s}\right\}$. Thus, it can be obtained that $\left|P^{i k}\right| \leq\left|Q^{j 0}\right|$ and $\left|P^{i 0}\right|<\left|Q^{j 0}\right|$. Hence, we have that

$$
\begin{aligned}
I E\left(C_{Q}\right) & =1-\frac{1}{|n|} \sum_{i=1}^{n} \frac{\left|Q^{i}\right|}{|U|} \\
& =1-\frac{1}{|n|}\left(\sum_{j=1, j \neq j 0}^{n} \frac{\left|Q^{j}\right|}{|U|}+\frac{\left|Q^{j 0}\right|}{|U|}\right) \\
& <1-\frac{1}{|n|}\left(\sum_{j=1, j \neq j 0}^{n} \frac{\left|Q^{j}\right|}{|U|}+\frac{1}{s} \sum_{k=1}^{s} \frac{\left|P^{i_{k}}\right|}{|U|}\right) \\
& \leq 1-\frac{1}{|m|} \sum_{i=1}^{m} \frac{\left|P^{i}\right|}{|U|} \\
& =\sum_{i=1}^{m} \frac{1}{|m|}\left(1-\frac{\left|P^{i}\right|}{|U|}\right)=\operatorname{IE}\left(C_{P}\right)
\end{aligned}
$$

i.e., $I E\left(C_{Q}\right)<I E\left(C_{P}\right)$. This completes the proof.

Proposition 3.13 shows that the information entropy with maximal consistent block nature of knowledge increases as the maximal consistent blocks become smaller through finer classification in incomplete information systems.

Proposition 3.14. Let $I I S$ be an incomplete information system with $P, Q \subseteq A$. If $P \preceq^{\prime} Q$, then $I E(Q) \leq I E(P)$.

Proof. Since $P \preceq^{\prime} Q$, it follows that for every $P^{i} \in C_{P}$, there exists $Q^{j} \in C_{Q}$ such that $P^{i} \subseteq Q^{j}$. Suppose $S_{P}(u)=$ $\{v \in U \mid(u, v) \in \operatorname{SIM}(P)\}$ and $S_{Q}(u)=\{v \in U \mid(u, v) \in$ $\operatorname{SIM}(Q)\}$, it follows from Property 4 in [32] that $S_{P}(u)$ $=\cup\left\{X_{k} \in C_{P} \mid X_{k} \subseteq S_{P}(u)\right\}=\cup\left\{X_{k} \in C_{P}(u)\right\}(k \leq m)$ and $S_{Q}(u)=\cup\left\{Y_{t} \in C_{Q} \mid Y_{t} \subseteq S_{Q}(u)\right\}=\cup\left\{Y_{t} \in C_{Q}(u)\right\}(t \leq n)$ in the view of maximal consistent block nature of knowledge. Then, it can be obtained that $u \in C_{P}(u), u \in$ $C_{Q}(u), u \notin C_{P}-C_{P}(u)$ and $u \notin C_{Q}-C_{Q}(u)$. Hence, it 
follows from $P \preceq^{\prime} Q$ that for any $X_{k} \in C_{P}(u)$, there exists $Y_{t} \in C_{Q}(u)$ such that $X_{k} \subseteq Y_{t}$. Thus, for any $u \in U$, we obtain that $S_{P}(u)=\cup\left\{X_{k} \in C_{P} \mid X_{k} \subseteq S_{P}(u)\right\}=\bigcup_{k=1}^{m} X_{k} \subseteq$ $\bigcup_{t=1}^{n} Y_{t}=\cup\left\{Y_{t} \in C_{Q} \mid Y_{t} \subseteq S_{Q}(u)\right\}=S_{Q}(u)$, that is to say, $\left|S_{P}(u)\right| \leq\left|S_{Q}(u)\right|$ for any $u \in U$. Therefore, $\sum_{i=1}^{|U|} \frac{1}{|U|}$ $\left(1-\frac{\left|S_{Q}\left(u_{i}\right)\right|}{|U|}\right) \leq \sum_{i=1}^{|U|} \frac{1}{|U|}\left(1-\frac{\left|S_{P}\left(u_{i}\right)\right|}{|U|}\right)$, i.e., $I E(Q) \leq I E(P)$. This completes the proof.

Proposition 3.14 states that the information entropy of knowledge increases as the maximal consistent blocks become smaller through finer classification in incomplete information systems. Then, in an $I I S$, it can be seen easily from the above proof that $S_{P}(u) \subseteq S_{Q}(u)$ for any $u \in U$ if $P \preceq$ ' $Q$, that is, the partial relation $P \preceq Q$ can be induced by the partial relation $P \preceq^{\prime} Q$. Therefore, it can be concluded that the partial relation $\preceq^{\prime}$ is a special instance of the partial relation $\preceq$ in incomplete information systems in fact.

\section{Information granulation-based uncertainty measures in incomplete information systems}

Information granulation is mainly used to study the uncertainty in rough set theory. In some sense, there exists a complement relation between entropy and information granulation [12]. In such a case, firstly information granulation and conditional information granulation are introduced to measure the uncertainty of incomplete information systems and incomplete decision systems respectively, secondly the complement relationships between information granulation and information entropy are investigated, thirdly a variant of the information granulation with maximal consistent block is presented to measure the uncertainty of incomplete information systems, and then some of their important properties are deduced.

\subsection{Information granulation and conditional information granulation in incomplete information systems}

Let $I I S$ be an incomplete information system, $U / \operatorname{SIM}(A)$ $=\left\{S_{A}\left(u_{1}\right), S_{A}\left(u_{2}\right), \cdots, S_{A}\left(u_{|U|}\right)\right\}$. Information granulation of knowledge $A$ in [10] is denoted by

$$
I G(A)=\sum_{i=1}^{|U|} \frac{\left|S_{A}\left(u_{i}\right)\right|}{|U|^{2}}
$$

where $\frac{\left|S_{A}\left(u_{i}\right)\right|}{|U|}$ represents the probability of tolerance class $S_{A}\left(u_{i}\right)$ within the universe $U$.

Property 4.1. Let $I I S$ be an incomplete information system. If $U / \operatorname{SIM}(A)=\delta$, then the information granulation of knowledge $A$ achieves the minimum value $I G(A)=0$. If $U / \operatorname{SIM}(A)=\omega$, then the information granulation of knowledge $A$ achieves the maximum value $I G(A)=1-\frac{1}{|U|}$. Clearly, for an $I I S$, we have that $0 \leq$ $I G(A) \leq 1-\frac{1}{|U|}$.

Proposition 4.1. Let $I I S$ be an incomplete information system. The relationship between the information granulation $I G(A)$ and the information entropy $I E(A)$ is as follows: $I G(A)+I E(A)=1$.

Proof. It follows immediately from Definition 3.1 that

$$
\begin{aligned}
I E(A) & =\sum_{i=1}^{|U|} \frac{1}{|U|}-\sum_{i=1}^{|U|} \frac{\left|S_{A}\left(u_{i}\right)\right|}{|U|^{2}}=1-\sum_{i=1}^{|U|} \frac{\left|S_{A}\left(u_{i}\right)\right|}{|U|^{2}} \\
& =1-I G(A),
\end{aligned}
$$

i.e., $I G(A)+I E(A)=1$. This completes the proof.

Definition 4.1. Let $I I S_{1}=(U, P), I I S_{2}=(U, Q)$ be two incomplete information systems. Joint information granulation of $P$ and $Q$ is defined as

$$
I G(P \cup Q)=\sum_{i=1}^{|U|} \frac{\left|S_{P}\left(u_{i}\right) \cap S_{Q}\left(u_{i}\right)\right|}{|U|^{2}} .
$$

Proposition 4.2. Let $I I S$ be an incomplete information system with $P, Q \subseteq A$. The relationship between the joint information granulation $I G(P \cup Q)$ and the joint information entropy $I E(P \cup Q)$ is as follows: $I G(P \cup Q)+$ $I E(P \cup Q)=1$.

Proof. The proof is similar to that of Proposition 4.1.

Propositions 4.1 and 4.2 show the relationship between the information granulation and the information entropy is the strict complement relationship, that is, they possess the same capability on depicting the uncertainty of an incomplete information system.

Corollary 4.1. Let $I I S$ be an incomplete information system with $P, Q \subseteq A$. Then $I G(P \cup Q)+I E(P \cup Q)=$ $I G(P)+I E(P)$.

Proof. It can be achieved by Propositions 4.1 and 4.2.

Definition 4.2. Let $I I S$ be an incomplete information system with $P, Q \subseteq A$. Conditional information granulation of $Q$ with respect to $P$ is defined as

$$
I G(Q \mid P)=\sum_{i=1}^{|U|} \frac{\left|S_{P}\left(u_{i}\right)\right|-\left|S_{P}\left(u_{i}\right) \cap S_{Q}\left(u_{i}\right)\right|}{|U|^{2}}
$$

Property 4.2. Let $I I S$ be an incomplete information system with $P, Q \subseteq A$. Then $I G(Q \mid P)=I E(Q \mid P)$.

In what follows, the proposition will establish the relationship between the information granulation and the conditional information granulation in an incomplete decision system.

Proposition 4.3. Let $I D S$ be an incomplete decision system with $P \subseteq C$. Then $I G(D \mid P)=I G(P)-I G(P \cup D)$ $=I E(P \cup D)-I E(P)=I E(D \mid P)$. 
Proof. It follows immediately from Definition 4.2 that

$$
\begin{aligned}
I G(D \mid P) & =\sum_{i=1}^{|U|} \frac{\left|S_{P}\left(u_{i}\right)\right|}{|U|^{2}}-\sum_{i=1}^{|U|} \frac{\left|S_{P}\left(u_{i}\right) \cap S_{D}\left(u_{i}\right)\right|}{|U|^{2}} \\
& =I G(P)-I G(P \cup D) .
\end{aligned}
$$

Then, from Property 4.2 and Proposition 3.7, it can be obtained that $I G(D \mid P)=I E(D \mid P)=I E(P \cup D)-I E(P)$. This completes the proof.

According to Proposition 4.3, it is known that the conditional information granulation is a special instance of the conditional entropy in incomplete decision systems. It means that the definition of conditional information granulation is a consistent extension to conditional entropy in incomplete decision systems. Therefore, they have the same capability on representing the uncertainty of an incomplete decision system.

\subsection{Information granulation with maximal consistent block in incomplete information systems}

Definition 4.3. Let $I I S$ be an incomplete information system, $P \subseteq A$, and $C_{P}=\left\{P^{1}, P^{2}, \cdots, P^{m}\right\}$. Information granulation of knowledge $P$ for the maximal consistent blocks $C_{P}$ is defined as

$$
I G\left(C_{P}\right)=\frac{1}{|m|} \sum_{i=1}^{m} \frac{\left|P^{i}\right|}{|U|},
$$

where $\frac{\left|P^{i}\right|}{|U|}$ represents the probability of maximal consistent block $P^{i}$ within the universe $U$.

Property 4.3. Let $I I S$ be an incomplete information system with $P \subseteq A$. If $C_{P}=\omega$, then the information granulation of knowledge $P$ achieves the minimum value $I G\left(C_{P}\right)=\frac{1}{|U|}$. If $C_{P}=\delta$, then the information granulation of knowledge $P$ achieves the maximum value $I G\left(C_{P}\right)=1$. Clearly, for an $I I S, \frac{1}{|U|} \leq I G\left(C_{A}\right) \leq 1$ holds.

Proposition 4.4. Let $I I S$ be an incomplete information system, $P, Q \subseteq A, C_{P}=\left\{P^{1}, P^{2}, \cdots, P^{m}\right\}$, and $C_{Q}=\left\{Q^{1}\right.$, $\left.Q^{2}, \cdots, Q^{n}\right\}$. If $P \prec^{\prime} Q$, then $I G\left(C_{P}\right)<I G\left(C_{Q}\right)$.

Proof. Suppose $P \prec^{\prime} Q$, it follows that for every $P^{i} \in C_{P}$, there exists $Q^{j} \in C_{Q}$ such that $P^{i} \subseteq Q^{j}$, and for some $P^{i 0} \in C_{P}$, there exists $Q^{j 0} \in C_{Q}$ such that $P^{i 0} \subset Q^{j 0}$ and $m>n$. It follows that for $Q^{j 0} \in C_{Q}$, there exists $\left\{P^{i 1}\right.$, $\left.P^{i 2}, \cdots, P^{i s}\right\}\left(P^{i k} \in C_{P}, k=\{1,2, \cdots, s\}\right)$ such that each $P^{i k} \subseteq Q^{j 0}$, where $P^{i 0} \in\left\{P^{i 1}, P^{i 2}, \cdots, P^{i s}\right\}$. Then, it can be obtained that $\left|P^{i k}\right| \leq\left|Q^{j 0}\right|$ and $\left|P^{i 0}\right|<\left|Q^{j 0}\right|$. Therefore, we can obtain that

$$
\begin{aligned}
I G\left(C_{Q}\right) & =\frac{1}{|n|}\left(\sum_{j=1, j \neq j 0}^{n} \frac{\left|Q^{j}\right|}{|U|}+\frac{\left|Q^{j 0}\right|}{|U|}\right) \\
& >\frac{1}{|n|}\left(\sum_{j=1, j \neq j 0}^{n} \frac{\left|Q^{j}\right|}{|U|}+\frac{1}{s} \sum_{k=1}^{s} \frac{\left|P^{i_{k}}\right|}{|U|}\right) \\
& >\frac{1}{|m|} \sum_{i=1}^{m} \frac{\left|P^{i}\right|}{|U|}=I G\left(C_{P}\right)
\end{aligned}
$$

i.e., $I G\left(C_{P}\right)<I G\left(C_{Q}\right)$. This completes the proof.

Proposition 4.4 shows that the information granulation with maximal consistent block nature of knowledge decreases as the maximal consistent blocks become smaller through finer classification in an incomplete information system.

Proposition 4.5. Let IIS be an incomplete information system with $P, Q \subseteq A$. If $P \preceq^{\prime} Q$, then $I G(P) \leq I G(Q)$.

Proof. The proof is similar to that of Proposition 3.14.

Proposition 4.5 shows that the information granulation decreases with the maximal consistent blocks becoming smaller through finer classification in incomplete information systems. In the following, we can establish the relationship between the information granulation and the information entropy with maximal consistent block in incomplete information systems.

Proposition 4.6. Let IIS be an incomplete information system with $P \subseteq A$. The relationship between the information granulation $I G\left(C_{P}\right)$ and the information entropy $I E\left(C_{P}\right)$ is as follows: $I G\left(C_{P}\right)+I E\left(C_{P}\right)=1$.

Proof. It follows from Definitions 3.6 and 4.3 that

$$
\begin{aligned}
I E\left(C_{P}\right) & =\sum_{i=1}^{m} \frac{1}{|m|}-\frac{1}{|m|} \sum_{i=1}^{m} \frac{\left|P^{i}\right|}{|U|}=1-\frac{1}{|m|} \sum_{i=1}^{m} \frac{\left|P^{i}\right|}{|U|} \\
& =1-I G\left(C_{P}\right),
\end{aligned}
$$

i.e., $I G\left(C_{P}\right)+I E\left(C_{P}\right)=1$. This completes the proof.

Proposition 4.6 states that in an incomplete information system, the relationship between the information granulation $I G\left(C_{A}\right)$ and the information entropy $I E\left(C_{A}\right)$ is the strict complement relationship. That is, they possess the same capability on depicting the uncertainty of incomplete information systems in the view of maximal consistent block nature of knowledge in incomplete information systems.

Remark. Unlike most of the existing measures for the uncertainty in incomplete information systems and incomplete decision systems, the relationships among these concepts (information entropy, conditional entropy, mutual information, information granulation, and conditional information granulation) can be established, which are formally expressed as $I E(Q \mid P)=I E(P \cup Q)-$ $I E(P), I E(P ; Q)=I E(P)-I E(P \mid Q)=I E(Q)-I E(Q \mid P)$, $I G(A)+I E(A)=1, I G(P \cup Q)+I E(P \cup Q)=1, I G(P \cup$ $Q)+I E(P \cup Q)=I G(P)+I E(P), I G(Q \mid P)=I E(Q \mid P)$, $I G\left(C_{P}\right)+I E\left(C_{P}\right)=1$ in an $I I S$ with $P, Q \subseteq A$, and 
$I G(D \mid P)=I G(P)-I G(P \cup D)=I E(P \cup D)-I E(P)=$ $I E(D \mid P)$ in an IDS with $P \subseteq C$. These relationships are very significant for reasonably applying an uncertainty measure to incomplete information systems and incomplete decision systems. However, most of the existing entropies and their extensions in incomplete information systems and incomplete decision systems can not establish the above relationships. Therefore, these uncertainty measures mentioned above may be a much better uncertainty measure for measuring the knowledge content of incomplete information systems and incomplete decision systems.

\section{Conclusions}

Real-world data sets are usually incomplete for various subjective and objective reasons; that is, they contain some missing (null) values. Rough set theory is a new mathematical tool for modeling uncertain or incomplete information. The development of a rough computational method is one of the most important research tasks. In this article, we introduce concepts of information entropy and information granulation-based uncertainty measures in incomplete information systems and incomplete decision systems, and based on maximal consistent block technique, the variants of the information entropy and the information granulation are presented to measure the discernibility ability of an information/knowledge in incomplete information systems. Then, some important properties of them are discussed. From these properties, it can be shown that these measures which are proposed provide important approaches to measuring the discernibility ability of different knowledge in incomplete information systems and incomplete decision systems. Furthermore, the relationships among these proposed measures are established as well. These results have a wide variety of applications, such as measuring the knowledge content and the significance of an attribute set, constructing a decision tree, and building a heuristic function in a heuristic reduct algorithm in incomplete information/decision systems. What's more, it is noted that these new measures also can be further extended to measure the uncertainty and may be helpful for rule evaluation and knowledge discovery in non-equivalencebased information systems.

\section{Acknowledgement}

This work was supported by the National Natural Science Foundation of China (Nos. 60873104, 61040037, 61370169), the Key Project of Science and Technology Department of Henan Province (No. 112102210194), the Science and Technology Research Key Project of Educational Department of Henan Province (Nos. 12A520027, 13A520529), the Education Fund for Youth
Key Teachers of Henan Normal University, and the Postgraduate Science and Technology Program of Beijing University of Technology (No. ykj-2012-6765).

The authors are grateful to the anonymous referee for a careful checking of the details and for helpful comments that improved this paper.

\section{References}

[1] Z. Pawlak and A. Skowron, Rudiments of rough sets, Information Sciences, 177, 3-27 (2007).

[2] L. Sun and J. C. Xu, A granular computing approach to gene selection, Bio-Medical Materials and Engineering, 24, 13071314 (2014).

[3] W. Z. Wu, Y. Leung and J. S. Mi, Granular computing and knowledge reduction in formal contexts, IEEE Transactions on Knowledge and Data Engineering, 21, 1461-1474 (2009).

[4] L. Sun, J. C. Xu, Y. W. Hu and L. N. Du, Granular space-based feature selection and its applications, Journal of Software, 8, 817-826 (2013).

[5] X. H. Zhang, B. Zhou and P. Li, A general frame for intuitionistic fuzzy rough sets, Information Sciences, 216, 3449 (2012).

[6] L. Sun, N. Zhong and J. C. Xu, Granularity-based user-centric multi-strategies and application in knowledge retrieval, Journal of Computers, 8, 2101-2109 (2013).

[7] F. Min, H. P. He, Y. H. Qian and W. Zhu, Test-cost-sensitive attribute reduction, Information Sciences, 181, 4928-4942 (2011).

[8] A. Mellit,H. Eleuch, M. Benghanem, C. Elaoun and A. Massi Pavan, Energy Conversion and Management, 51, 771-782 (2010).

[9] L. Sun, J. C. Xu and Y. Tian, Feature selection using rough entropy-based uncertainty measures in incomplete decision systems, Knowledge-Based Systems, 36, 206-216 (2012).

[10] J. Y. Liang and D. Y. Li, Uncertainty and Knowledge Acquisition in Information Systems, Science Press, Beijing, (2005).

[11] L. Sun, J. C. Xu, S. Q. Li, X. Z. Cao and Y. P. Gao, New approach for feature selection by using information entropy, Journal of Information and Computational Science, 8, 15251532 (2011).

[12] J. Y. Liang and Y. H. Qian, Information granules and entropy theory in information systems, Science in China Series F: Information Sciences, 51, 1427-1444 (2008).

[13] L. Sun, J. C. Xu, J. Y. Ren, T. H. Xu and Q. Q. Zhang, Granularity partition-based feature selection and its application in decision systems, Journal of Information and Computational Science, 9, 3487-3500 (2012).

[14] C. E. Shannon, The mathematical theory of communication, The Bell System Technical Journal, 27, 373-423 (1948).

[15] Claude Gravel, Quantum Physics Letters, 1, 87-96 (2012).

[16] K. Berrada, H Eleuch and Y. Hassouni, J. Phys. B: At. Mol. Opt. Phys., 44, 145503 (2011).

[17] Kamal Berrada, Abderrahim Chafik, Hichem Eleuch and Yassine Hassouni, Quantum Inf. Process., 9, 13-26 (2010).

[18] K. Berrada , M. El Baz, H. Eleuch and Y. Hassouni, International Journal of Modern Physics C,21, 291-305 (2010). 
[19] L. Sun, J. C. Xu, Z. A. Xue and L. J. Zhang, Rough entropy-based feature selection and its application, Journal of Information and Computational Science, 8, 1525-1532 (2011).

[20] T. Beaubouef, F. E. Petry and G. Arora, Informationtheoretic measures of uncertainty for rough sets and rough relational databases, Information Sciences, 109, 185-195 (1998).

[21] E. Hernandez and J. Recasens, A reformulation of entropy in the presence of indistinguishability operators, Fuzzy Sets and Systems, 128, 185-196 (2002).

[22] D. Q. Miao and J. Wang, On the relationships between information entropy and roughness of knowledge in rough set theory, Pattern Recognition and Artificial Intelligence, 11, 34-40 (1998).

[23] J. Y. Liang, K. S. Chin, C. Y. Dang and C. M. YAM, A new method for measuring uncertainty and fuzziness in rough set theory, International Journal of General Systems, 31, 331-342 (2002).

[24] J. S. Mi, Y. Leung and W. Z. Wu, An uncertainty measure in partition-based fuzzy rough sets, International Journal of General Systems, 34, 77-90 (2005).

[25] B. W. Xu, Y. M. Zhou and H. M. Lu, An improved accuracy measure for rough sets, Journal of Computer and System Sciences, 71, 163-173 (2005).

[26] L. A. Zadeh, Fuzzy sets and information granularity, in Advances in Fuzzy Set Theory and Application, NorthHolland, Amsterdam, 3-18 (1979).

[27] L. Sun, J. C. Xu, C. Wang, T. H. Xu and J. Y. Ren, Granular computing-based granular structure model and its application in knowledge retrieval, Information Technology Journal, 11, 1714-1721 (2012)

[28] L. Sun, J. C. Xu, Z. A. Xue and J. Y. Ren, Decision degreebased decision tree technology for rule extraction, Journal of Computers, 7, 1769-1779 (2012).

[29] J. C. Xu and L. Sun, A new knowledge reduction algorithm based on decision power in rough set, Transactions on Rough Sets, 12, 76-89 (2010).

[30] J. Y. Liang, Z. Z. Shi and D. Y. Li, Information entropy, rough entropy and knowledge granulation in incomplete information systems, International Journal of General Systems, 35, 641-654 (2006).

[31] M. J. Wierman, Measuring uncertainty in rough set theory, International Journal of General Systems, 28, 283-297 (1999).

[32] Y. Leung and D. Y. Li, Maximal consistent block technique for rule acquisition in incomplete information systems, Information Sciences, 153, 85-106 (2003).

[33] J. C. Xu and L. Sun, Knowledge entropy and feature selection in incomplete decision systems, Applied Mathematics and Information Sciences, 6, 829-837 (2013).

[34] Y. Y. Yao, Relational interpretations of neighborhood operators and rough set approximation operators, Information Sciences, 101, 239-259 (1998).

[35] L. Sun and J. C. Xu, Gene selection using mutual information based uncertainty measures for tumor classication, Bio-Medical Materials and Engineering, 24, 763-770 (2014).

[36] Q. H. Hu and D. R. Yu, Entropies of fuzzy indiscernibility relation and its operations, International Journal of Uncertainty, Fuzziness and Knowledge-Based Systems, 12, 575-589 (2004).
[37] W. H. Xu and W. X. Zhang, Measuring roughness of generalized rough sets induced by a covering, Fuzzy Sets and Systems, 158, 2443-2455 (2007).

[38] B. Huang, X. He and X. Z. Zhou, Rough entropy based on generalized rough set covering reduction, Chinese Journal of Software, 15, 215-220 (2004).

[39] J. Y. Liang and Z. B. Xu. The algorithm on knowledge reduction in incomplete information systems, International Journal of Uncertainty, Fuzziness and Knowledge-Based Systems, 24, 95-103 (2002).

[40] Y. H. Qian, J. Y. Liang and F. Wang, A new method for measuring the uncertainty in incomplete information systems, International Journal of Uncertainty, Fuzziness and Knowledge-Based Systems, 17, 855-880 (2009).

[41] D. R. Yu, Q. H. Hu and C. X. Wu, Uncertainty measures on fuzzy relations and their applications, Applied Soft Computing, 7, 1135-1143 (2007).

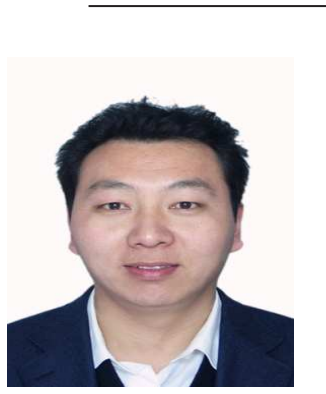

Lin Sun works at College of Computer \& Information Engineering, Henan Normal University. $\mathrm{He}$ is currently a Ph.D. Candidate in School of Electronic Information and Control Engineering, Beijing University of Technology. $\mathrm{He}$ received his B.S. and M.S. degree in Computer Science and Technology, Henan Normal University in 2003 and 2007, respectively. His main research interests include granular computing, rough set, and data mining.

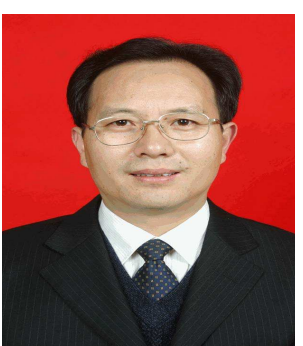

Jiucheng $\mathbf{X u}$ is currently a Professor at College of Computer \& Information Engineering, Henan Normal University. He received his B.S. degree in Mathematics, Henan Normal University in 1986, the M.S. degree and the Ph.D. degree in Computer Science and Technology, Xi' an Jiaotong University in 1995 and 2004, respectively. His main research interests include granular computing, rough set, data mining, intelligent information processing, and bioinformatics.

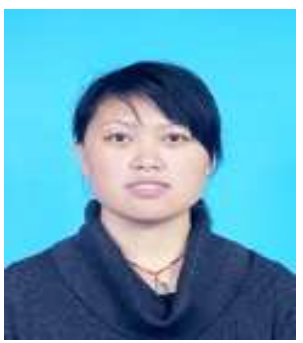

Tianhe $\mathbf{X u}$ is currently a postgraduate in College of Computer \& Information Engineering, Henan Normal University. She received her B.S. degree in Educational Technology, Luoyang Normal University in 2011. Her main research interests include granular computing, data mining,

and bioinformatics. 